\title{
Combined Antiretroviral Therapy Causes Cardiomyopathy and Elevates Plasma Lactate in Transgenic AIDS Mice
}

\author{
William Lewis, Chad P. Haase, Scott M. Raidel, Rodney B. Russ, Roy L. Sutliff, \\ Brian D. Hoit, and Allen M. Samarel
}

Department of Pathology (WL, CPH, SMR, RBR, RLS), Emory University School of Medicine, Atlanta, Georgia; University Hospital Cleveland (BDH), Cleveland, Ohio; and Cardiovascular Institute (AMS), Stritch School of Medicine, Loyola University Chicago, Maywood, Illinois

\begin{abstract}
SUMMARY: Highly active antiretroviral therapy (HAART) is implicated in cardiomyopathy (CM) and in elevated plasma lactate (LA) in AIDS through mechanisms of mitochondrial dysfunction. To determine mitochondrial events from HAART in vivo, 8-week-old hemizygous transgenic AIDS mice (NL4-3A gag/pol; TG) and wild-type FVB/n littermates were treated with the HAART combination of zidovudine, lamivudine, and indinavir or vehicle control for 10 days or 35 days. At termination of the experiments, mice underwent echocardiography, quantitation of abundance of molecular markers of CM (ventricular mRNA encoding atrial natriuretic factor [ANF] and sarcoplasmic calcium ATPase [SERCA2]), and determination of plasma LA. Myocardial histologic features were analyzed semiquantitatively and results were confirmed by transmission electron microscopy. After 35 days in the TG + HAART cohort, left ventricular mass increased $160 \%$ by echocardiography. Molecularly, ANF mRNA increased $250 \%$ and SERCA2 mRNA decreased $57 \%$. Biochemically, LA was elevated ( $8.5 \pm 2.0 \mathrm{~mm})$. Pathologically, granular cytoplasmic changes were found in cardiac myocytes, indicating enlarged, damaged mitochondria. Findings were confirmed ultrastructurally. No changes were found in other cohorts. After 10 days, only ANF was elevated, and only in the TG + HAART cohort. Results show that cumulative HAART caused mitochondrial CM with elevated LA in AIDS transgenic mice. (Lab Invest 2001, 81:1527-1536).
\end{abstract}

$Z$ idovudine (AZT; 3'-azido-2', 3'-deoxythymidine) was the first nucleoside reverse transcriptase inhibitor (NRTI) to treat HIV-1 infection and AIDS (Chaisson et al, 1986; Yarchoan et al, 1986). Monotherapy with AZT is uncommon in the United States today because highly active antiretroviral therapy (HAART) is a formidable clinical combination. HAART typically includes two NRTI plus an HIV-1 protease inhibitor (DHHS, 2001).

AZT caused a mitochondrial skeletal myopathy (Arnaudo et al, 1991; Dalakas et al, 1990). Characteristic microscopic "ragged red fibers" (Shoubridge, 1994) and ultrastructural paracrystalline inclusions occurred. A high lactate (LA)/pyruvate ratio (consistent with abnormal mitochondrial function) was seen in blood of patients with AZT mitochondrial myopathy (Chariot et al, 1994).

Analogous to AZT-induced mitochondrial skeletal myopathy in humans, cardiomyopathy (CM) and mitochondrial skeletal myopathy occur in AZT-treated rats (Corcuera Pindado et al, 1994; Lamperth et al, 1991; Lewis et al, 1991, 1992) and transgenic (TG) AIDS mice (NL4-3s gag/pol; Lewis et al, 2000). A relation-

Received July 20, 2001.

This work was supported by NIH HL59798 from NHLBI (WL).

Address reprint requests to: Dr. William Lewis, Department of Pathology, Emory University School of Medicine, 7117 WoodruffMemorial Building, 1639 Pierce Drive, Atlanta, GA 30322.E-mail:wlewis@emory.edu ship exists between the development of AIDS CM in adults and prolonged treatment with AZT (Barbaro et al, 1998, 2000; Herskowitz et al, 1992). The mechanism of CM from AZT is not completely understood, but may relate to altered mtDNA replication, its resultant effects on energetics, and related cellular processes (Lewis, 2000).

Recently, lactic acidemia in AIDS gained attention as an important clinical problem found both in adults (Boubaker et al, 2000; Lonergan et al, 2000; Ter Hofstede et al, 2000) and in children (Church et al, 2000). Treatment with certain NRTI (stavudine, lamivudine) resulted in anion gap acidosis (Miller et al, 2000; Moore et al, 2000).

To determine the effects of HAART on myocardial and mitochondrial structure and function in AIDS, a $2 \times 2$ protocol employed 8-week-old TG AIDS mice (NL4-3s gag/pol) and wild-type (WT) FVB/n littermates with and without HAART treatment. HAART included zidovudine, lamivudine, and indinavir. Treatment duration was 10 days and 35 days, respectively.

Molecular markers of cardiac remodeling, echocardiographic evidence of left ventricular enlargement, microscopic and ultrastructural features of mitochondrial CM, and elevated plasma LA were found after 35 days, but only in the TG + HAART cohort. After 10 days, only atrial natriuretic factor (ANF) elevation occurred, and only in the TG + HAART cohort. Together, these data indicate that HAART causes cumulative 
mitochondrial CM and elevated LA in AIDS that follows duration of HAART treatment.

\section{Results}

\section{General}

TG and WT mice each consumed AZT and lamivudine (3TC) in drinking water. For 35-day protocols, cumulative consumption (TG and $\mathrm{WT}$, respectively) was $141 \pm 7$ and $113 \pm 5 \mathrm{mg}$ for AZT; $7.2 \pm 0.3$ and $5.8 \pm$ $0.3 \mathrm{mg}$ for $3 \mathrm{TC}$; and $32 \pm 0.2$ and $32 \pm 0.2 \mathrm{mg}$ for indinavir. For the 10-day protocols, consumption (TG and WT, respectively) was $50 \pm 3$ and $39 \pm 1 \mathrm{mg}$ for $\mathrm{AZT} ; 2.6 \pm 0.1$ and $2.0 \pm 0.1 \mathrm{mg}$ for $3 \mathrm{TC}$; and $9 \pm 0.1$ and $9 \pm 0.1 \mathrm{mg}$ for indinavir (Table 1 ).

\section{Echocardiographic Findings}

M-mode echocardiography was performed and evaluated on TG and WT mice with and without HAART. Left ventricular (LV) mass was calculated for both 35-day and 10-day treatment protocols.

Figure $1 \mathrm{~A}$ is a collage of representative M-mode echocardiograms taken at midventricular level of hearts of WT + vehicle (upper left), WT + HAART (lower left), TG + vehicle (upper right), and TG + HAART (lower right) mice after the 35-day treatment. Significant LV thickening and dilation was found in the TG + HAART cohort.

LV mass was quantified after treatment and striking differences were found among the cohorts (Fig. 1B). As shown in the histogram, after the 35-day treatment, LV mass (normalized to body weight; $\mathrm{mg} / \mathrm{g}$ ) was $2.16 \pm 0.22$ in TG + HAART, $1.32 \pm 0.07$ in WT + HAART, $1.47 \pm 0.15$ in TG + vehicle, and $1.15 \pm 0.07$ in WT + vehicle cohorts. Normalized LV mass increased $160 \%$ above the WT + vehicle controls, and $>130 \%$ in the TG + HAART cohort compared with either WT + HAART or TG + vehicle $(p<0.001$ for each comparison; Fig. 1B). In contrast to the 35-day treatment protocols, after HAART treatment for 10 days, echocardiographic changes were absent in the cohorts (Table 2).

\section{Molecular Studies From Hearts of 35-day and 10-day Treatment Protocols}

Steady-state abundance of mRNA encoding ANF and sarcoplasmic calcium ATPase (SERCA2) were determined by Northern blot analysis of ventricular tissue.
Representative Northern blots (two samples from each cohort) from the 35-day protocol are presented as a collage in Figure 2A. No difference in ANF mRNA signal was found in cardiac ventricular samples obtained from WT + HAART or TG + vehicle when radioactive signals were compared with those obtained from samples from the WT + vehicle cohort. In contrast, a profound effect on abundance of ventricular ANF mRNA was seen in samples from the TG + HAART cohort (Fig. 2A). Quantitative analysis of cardiac ventricular ANF mRNA abundance revealed a 2.5-fold increase in the TG + HAART cohort compared with untreated controls ( $p<0.05$; Fig. 2B).

Reciprocal changes were observed in the abundance of SERCA2 mRNA. As seen in Figure 2, SERCA2 mRNA levels in TG + vehicle were $56 \pm 16 \%$ of those values from WT + vehicle ( $p<0.05$; Fig. $2 B$ ). This effect of TG expression per se on ventricular SERCA2 abundance was reported previously (Lewis et al, 2000). HAART treatment further reduced SERCA2 mRNA abundance in the TG animals to $43 \pm$ $7 \%$ of the level observed in untreated, WT animals.

In the HAART 10-day treatment experiment, similar alterations in ANF mRNA abundance (a sensitive index of cardiac molecular changes) were observed in the TG + HAART cohort; however, there were no significant differences in SERCA2 mRNA expression among the different cohorts (data not shown).

\section{Plasma LA}

Plasma LA was determined in all cohorts in both the 35-day and 10-day protocols. After 35 days (Fig. 3), samples from the TG + HAART cohort revealed strikingly elevated plasma LA $(8.5 \pm 2.0 \mathrm{~mm})$ compared with values from WT + HAART $(3.0 \pm 0.4 \mathrm{~mm}), \mathrm{TG}+$ vehicle $(1.6 \pm 0.5 \mathrm{~mm})$, and $\mathrm{WT}+$ vehicle $(2.7 \pm 0.5$ $\mathrm{mm})$. The difference was significant for the TG + HAART cohort compared with the others $(p<0.001)$. No differences in mean plasma LA values were found in samples from the 10-day HAART protocol (Table 3). Mean plasma LA values ranged from 1.3 to $4.0 \mathrm{~mm}$ after the 10-day treatment.

\section{Histopathologic Changes}

Samples taken from the 35-day experiment revealed differences in the microscopic structure of the myocardium. Samples from the TG + HAART cohort at 35 days exhibited numerous cardiac myocytes with gran-

Table 1. Cumulative Consumption of Antiretrovirals

\begin{tabular}{|c|c|c|c|c|}
\hline \multirow[b]{2}{*}{ Agent } & \multicolumn{2}{|c|}{35 days } & \multicolumn{2}{|c|}{10 days } \\
\hline & $\mathrm{TG}$ & WT & $\mathrm{TG}$ & WT \\
\hline AZT & $141 \pm 7^{*}$ & $113 \pm 5$ & $50 \pm 3^{* *}$ & $39 \pm 1$ \\
\hline 3TC & $7.2 \pm 0.3$ & $5.8 \pm 0.3$ & $2.6 \pm 0.1$ & $2.0 \pm 0.1$ \\
\hline Indinavir & $32 \pm 0.2$ & $32 \pm 0.2$ & $9 \pm 0.1$ & $9 \pm 0.1$ \\
\hline
\end{tabular}

AZT, zidovudine; 3TC, lamivudine; TG, transgenic mice; WT, wild-type mice.

Data are mean \pm SEM $(\mathrm{mg})$.

${ }^{*} p<0.001$ compared with WT at 35 days; ${ }^{* *} p<0.05$ compared with WT at 10 days. 
A

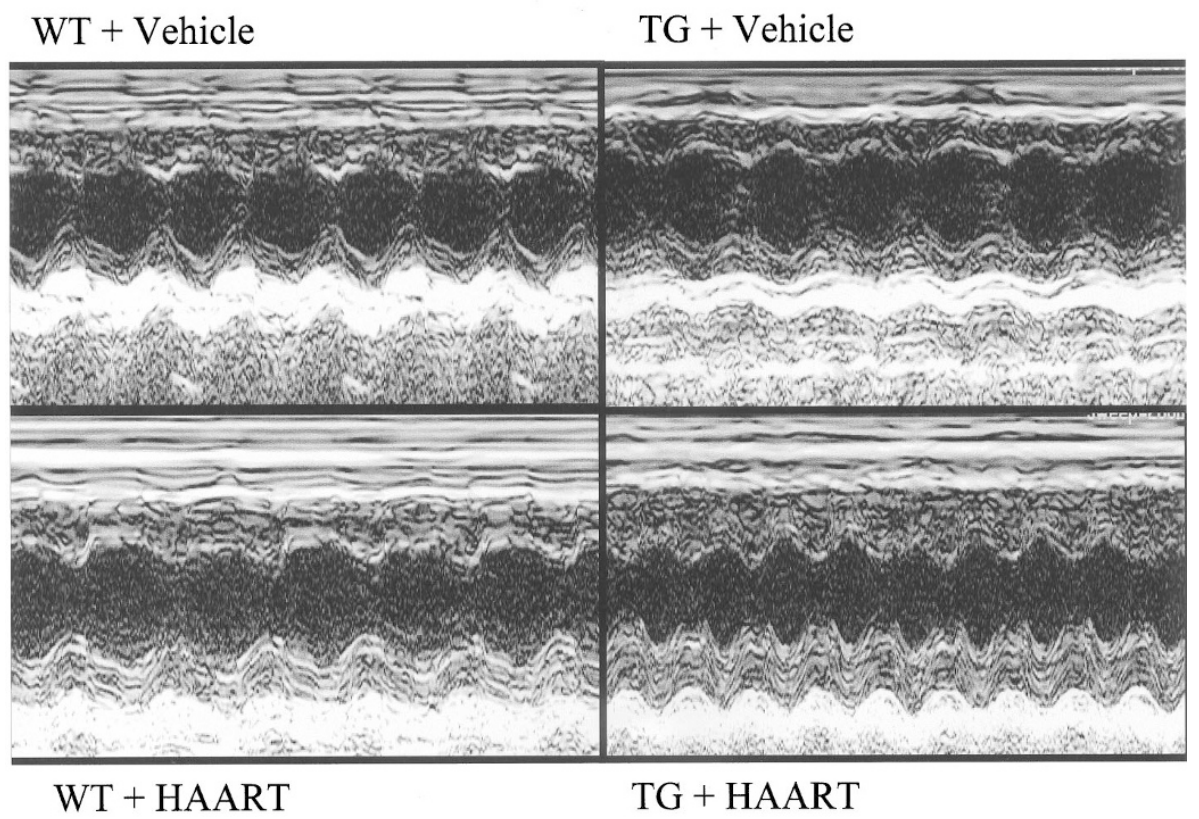

$\mathrm{B}$

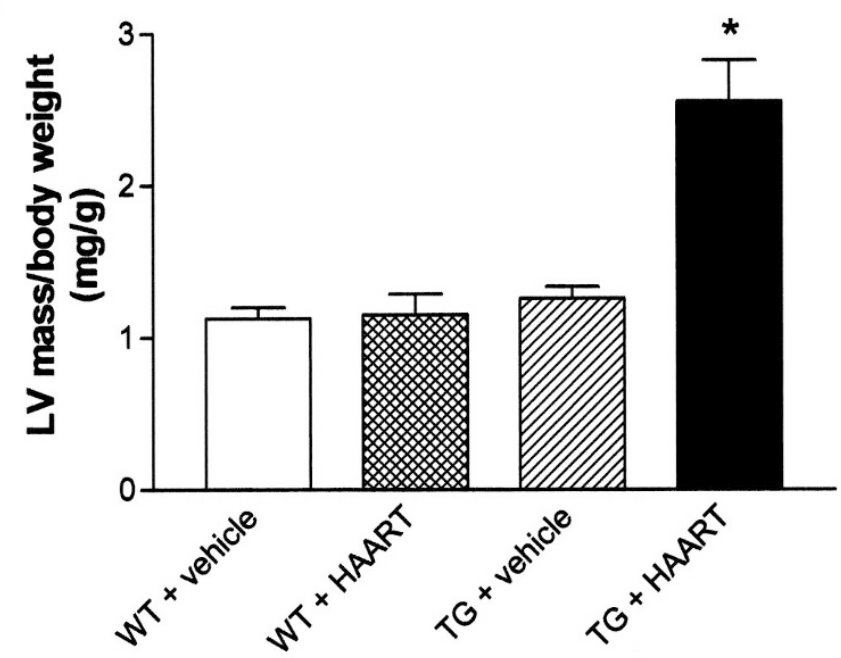

* $p<0.001$ compared to all other groups

Figure 1.

Echocardiography of transgenic (TG) and wild-type (WT) mice with highly active antiretroviral therapy (HAART) for 35 days. A, Representative M-mode echocardiograms taken at the midventricular level of WT + vehicle (upper left), WT + HAART (lower left), TG + vehicle (upper right), TG + HAART (lower right). TG + HAART demonstrates ventricular thickening and dilation after 35 days compared with all other cohorts. B, Histogram of calculated left ventricular (LV) mass. LV mass was calculated in a blinded fashion, code was broken, and data tabulated. Data are normalized to body weight $(\mathrm{mg} / \mathrm{g})$ and plotted as mean \pm SEM. The TG + HAART cohort reveals profoundly increased LV mass after 35 days $(p<0.001)$.

ular cytoplasm (Fig. 4) compared with the samples from the other cohorts at 35 days. Neither interstitial inflammation nor fibrosis was found.

Semiquantitative analysis of cardiac myocyte granularity was performed on scored samples (Fig. 5). Cytoplasmic granularity was a reliable marker of cardiac pathologic change in the TG + HAART cohort $(p<0.05)$. This morphologic feature correlated with biochemical, molecular, and echocardiographic findings in the TG + HAART cohort treated for 35 days (above). Microscopic sections from the 10-day HAART treatment cohorts revealed no differences in granularity of cardiac myocytes (data not shown).

\section{Transmission Electron Microscopy}

Ultrastructural changes in cardiac myocytes were determined in the 35-day experiment. Ultrastructural findings paralleled and supported the histopathologic studies. Mitochondria were damaged in cardiac myocytes in the TG + HAART cohort. Pathologic features 
Table 2. Echocardiographic Measurements

\begin{tabular}{lcccc}
\hline Measurement & TG + HAART & WT + HAART & TG + Vehicle & WT + Vehicle \\
\hline 35-day HAART & & & & \\
LVEDD & $3.94 \pm 0.14$ & $3.64 \pm 0.04$ & $3.65 \pm 0.13$ & $3.4 \pm 0.06$ \\
PWTH & $0.35 \pm 0.01$ & $0.32 \pm 0.01$ & $0.31 \pm 0.01$ & $0.3 \pm 0.004$ \\
R-R & $161.8 \pm 12.4$ & $150.3 \pm 6.1$ & $156.4 \pm 8.2$ & $154.4 \pm 7.7$ \\
LV Mass & $41.8 \pm 4.4$ & $31.0 \pm 1.2$ & $31.0 \pm 3.7$ & $26.2 \pm 1.1$ \\
LV Mass (mg/g) & $2.16 \pm 0.22^{*}$ & $1.32 \pm 0.07$ & $1.47 \pm 0.15$ & $1.15 \pm 0.07$ \\
10-day HAART & & & & \\
LVEDD & $3.32 \pm 0.19$ & $3.51 \pm 0.13$ & $3.51 \pm 0.15$ & $3.32 \pm 0.09$ \\
PWTH & $0.37 \pm 0.01$ & $0.35 \pm 0.01$ & $0.36 \pm 0.03$ & $0.33 \pm 0.01$ \\
R-R (MS) & $156.2 \pm 17.0$ & $142.1 \pm 7.0$ & $245.7 \pm 71.6$ & $153.6 \pm 7.1$ \\
LV Mass & $32.0 \pm 3.4$ & $32.8 \pm 2.8$ & $35.1 \pm 6.1$ & $27.6 \pm 1.5$ \\
LV Mass (mg/g) & $1.44 \pm 0.13$ & $1.34 \pm 0.12$ & $1.5 \pm 0.15$ & $1.19 \pm 0.06$ \\
\hline
\end{tabular}

HAART, highly active antiretroviral therapy; LVEDD, left ventricular end diastolic dimension; PWTH, posterior wall thickness; R-R, interval between two successive heartbeats (in milliseconds).

Data are mean \pm SEM.

${ }^{*} p<0.001$ compared with all groups.

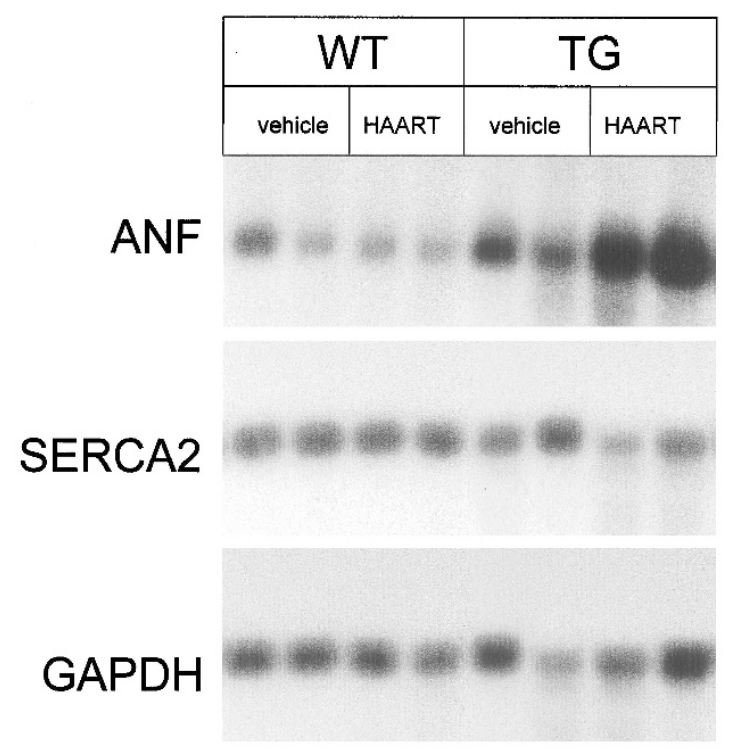

A
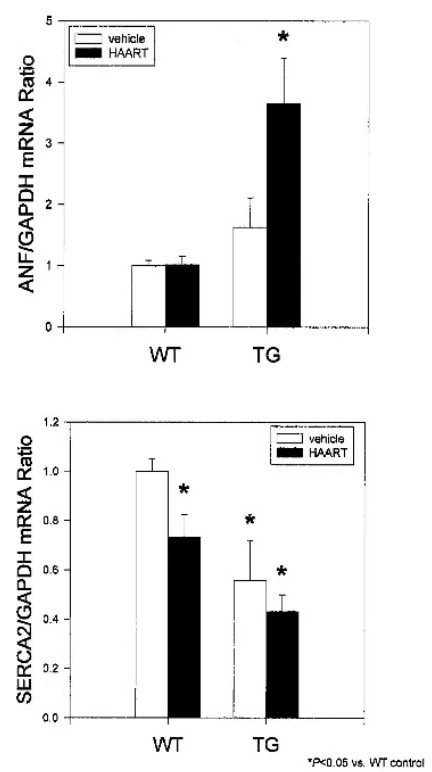

B

Figure 2.

Cardiac mRNA in TG and WT mice. Steady-state abundance of mRNA encoding atrial natriuretic factor (ANF), sarcoplasmic calcium ATPase (SERCA2), and glyceraldehyde phosphate dehydrogenase (GAPDH) (top to bottom) were determined in cardiac samples from mice in the 35-day HAART protocol. A, Representative Northern blots for WT + vehicle, WT + HAART, TG + vehicle, and TG + HAART cohorts are presented from two animals in each cohort. B, Histogram of data obtained from all animals in each cohort. Steady-state abundance of ANF RNA increased 250\% in the TG + HAART cohort compared with WT or WT + HAART and compared with TG + vehicle $(p<0.001$ for each) (B). After 35 days, SERCA2 mRNA abundance decreased significantly in the TG + HAART cohort compared with untreated WT mice ( $43 \pm 7 \%$ decrease) (B).

included mitochondrial swelling and fragmentation of cristae (Fig. 6).

\section{Discussion}

Cardiac mitochondrial dysfunction was manifested as molecular, structural, and functional changes of $\mathrm{CM}$ and elevated plasma LA in AIDS TG mice treated with HAART for 35 days. The regimen consisted of two $\mathrm{NRTI}$ plus indinavir. Echocardiographic, molecular, pathologic, and biochemical evidence support the diagnosis and indicate mitochondrial dysfunction plays a key role in $\mathrm{CM}$ development.
Data from these experiments allow two important conclusions to be drawn. First, compared with monotherapy with AZT, treatment with two NRTI plus a protease inhibitor resulted in worse cardiac mitochondrial toxicity that included CM with elevated LA. Second, HAART treatment of TG mice yielded profound LV changes after 35 days, but not after 10 days, when only ANF mRNA abundance was elevated. This suggested a cumulative effect of HAART on the development of mitochondrial dysfunction.

A pharmacologic relationship occurred between CM development, elevated plasma LA, and total HAART 


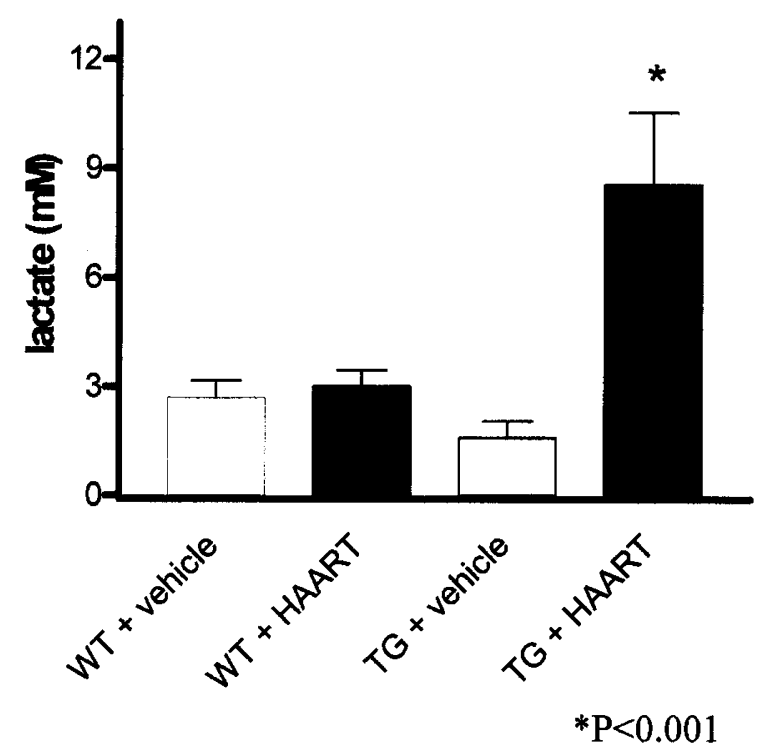

Figure 3.

Histogram of plasma lactate levels in TG and WT mice treated with HAART or vehicle for 35 days. Plasma lactate levels were determined colorimetrically in all cohorts. Samples from the TG + HAART cohort reveal elevated plasma lactate (mean $=8.5 \pm 2.0 \mathrm{~mm} ; n=8$ ) compared with values from the WT + HAART cohort $(3.0 \pm 0.4 \mathrm{~mm} ; n=12)$, the TG + vehicle cohort $(1.6 \pm 0.5$ $\mathrm{mm} ; n=4)$, and the WT + vehicle cohort $(2.7 \pm 0.5 \mathrm{~mm} ; n=11)$. The difference is significant for each group compared with TG + HAART ( $p<$ 0.001).

consumption over 35 days. Of the parameters examined, only elevated cardiac ventricular ANF mRNA was found after 10 days, and only in the TG + HAART cohort. Based on current HAART regimens for adult humans (DHHS, 2001), NRTI doses for mice were somewhat higher $(\mathrm{mg} / \mathrm{kg} / \mathrm{d})$. Indinavir doses resembled doses for humans.

Although it is possible to conclude that a relatively high AZT dose was employed experimentally in mice, doses are not always comparable between species. Similar (or even higher) AZT doses were employed in other monotherapy studies in vivo (Lamperth et al, 1991; Lewis et al, 1991, 1992, 2000; Ruprecht et al, 1986). CM was more severe in the present HAART experiments than reported previously (Lewis et al, 1991, 2000; McCurdy and Kennedy, 1998), in which higher AZT doses were used. Data suggest that development of mitochondrial CM depends on cumulative HAART.

HAART cardiac mitochondrial toxicity was documented echocardiographically. A significant increase in LV mass $(+160 \%)$ was found in the TG + HAART cohort after 35 days. This point was corroborated by pathologic, biochemical, and molecular findings.

Molecular correlates of mitochondrial CM were striking in myocardial samples obtained from the TG + HAART cohort after 35 days. Molecular changes in the cardiac ventricle included increased steady-state abundance of mRNA encoding ANF and decreased mRNA encoding SERCA2, two important markers of $\mathrm{CM}$ in vivo (Hunter and Chien, 1999). After 10 days, only ANF mRNA abundance was elevated in the TG + HAART cohort. It is possible to suggest that increased abundance of ANF mRNA in the TG + HAART cohort at 10 days may have served as a predictor of cardiac molecular changes of $\mathrm{CM}$ that were present at 35 days in the analogous cohort (with increased duration of HAART).

As seen in Figure 2, ANF mRNA levels tended to be higher in TG, vehicle-treated animals than in respective WT mice, whereas HAART led to a further, significant increase in the level of its expression. Upregulation of ANF gene expression in ventricular myocytes was accompanied by a profound decrease in SERCA2 mRNA levels, which was further reduced by HAART treatment. In a previous study from our laboratories (Lewis et al, 2000), we showed that ANF mRNA levels were increased, and SERCA2 mRNA levels were decreased in TG male animals even in the absence of antiretroviral treatment. These reciprocal alterations in gene expression have been found to be sensitive indices of cardiac hypertrophy and failure in both experimental animals and humans with cardiomyopathies of diverse etiology. Indeed, these molecular changes were closely associated with a significant impairment in systolic and diastolic function in TG mice (Lewis et al, 2000). We suspect that these molecular alterations may be important in clinical practice because they may indicate that HIV predisposes affected individuals to further mitochondrial damage induced by retroviral therapy.

Pathologic findings and semiquantitative analyses supported the diagnosis of CM at the cellular level. Granularity of cardiac myocyte cytoplasm was found in the TG + HAART cohort after 35 days. Although not directly addressed in the present study, granularity of cardiac myocyte cytoplasm may correlate with altered mitochondrial biogenesis and CM (Lehman et al, 2000). Classic "ragged red fibers" are found in striated muscle samples from patients with certain mitochondrial encephalomyopathies and in AZT skeletal myopathy (Arnaudo et al, 1991; Dalakas et al, 1990). They correlate with ultrastructural features of mitochondrial damage and proliferation (Shoubridge, 1994). In the present study, cardiac myocyte cytoplasmic granularity was a correlative feature of ultrastructural evidence of cardiac mitochondrial injury.

Ultrastructural findings bolstered the molecular, histopathologic, and echocardiographic data above. Mitochondrial ultrastructural damage was found in HAART-treated TG mice after 35 days. The damage resembled the damage with AZT monotherapy in the rat and TG mice (Lamperth et al, 1991; Lewis et al, 1991, 2000).

Elevated LA in plasma samples was a noteworthy biochemical derangement that accompanied CM in the $\mathrm{TG}+\mathrm{HAART}$ cohort at 35 days. It is reasonable to suggest that elevated plasma $L A$ relates to inadequate oxidative metabolism. Myocardium and other tissues may participate in the metabolic derangement. Elevated plasma LA (particularly in the presence of acidemia) is an ominous clinical event. Lactic acidosis per se has detrimental effects on cardiac performance (Adrogue and Madias, 1998). If it is reasonable to consider elevated plasma LA an indicator of worse prognosis, its presence 
Table 3. Plasma Lactate with HAART

\begin{tabular}{ccccc}
\hline $\begin{array}{c}\text { Protocol } \\
\text { Duration }\end{array}$ & WT + Vehicle & WT + HAART & TG + Vehicle \\
$(\mathrm{n})$ & $(\mathrm{n})$ & $\begin{array}{c}\text { TG }+ \text { HAART } \\
(\mathrm{n})\end{array}$ \\
\hline 35 days & $2.7 \pm 0.5$ & $3.0 \pm 0.4$ & $1.6 \pm 0.6$ & $8.5 \pm 2.0^{*}$ \\
& $(11)$ & $(12)$ & $(4)$ & $(8)$ \\
10 days & $2.5 \pm 0.3$ & $1.3 \pm 0.2$ & $4.0 \pm 0.8$ & $4.0 \pm 0.6$ \\
& $(9)$ & $(9)$ & $(6)$ & $(9)$ \\
\hline
\end{tabular}

Data are mean \pm SEM $(\mathrm{nm})$

${ }^{*} p<0.001$ compared to 35-day cohorts
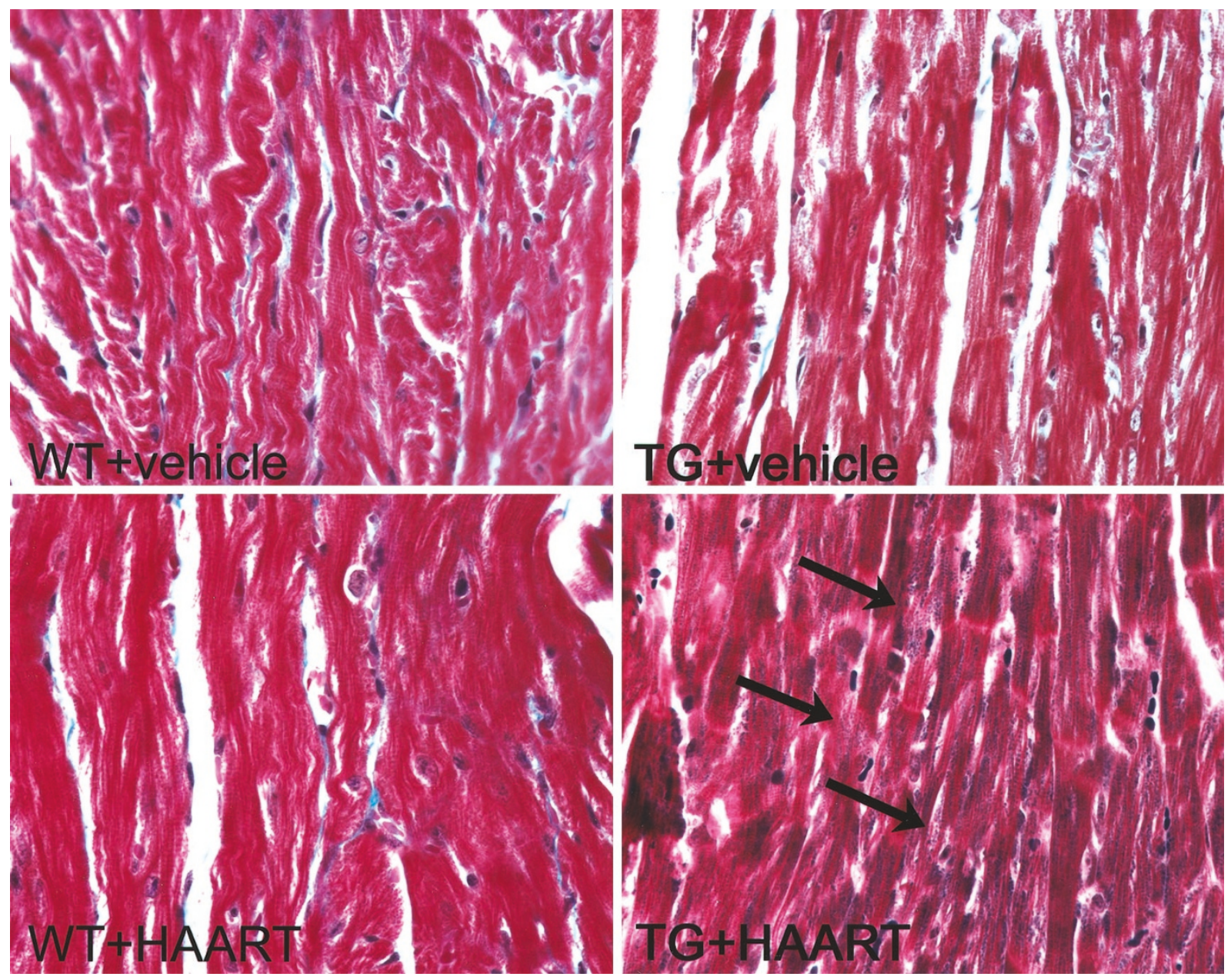

Figure 4.

Histopathologic changes and semiquantitative analysis of myocyte granularity in myocardial sections. Cross-sectional slices of myocardium from WT + vehicle (upper left), WT + HAART (lower left), TG + vehicle (upper right), and TG + HAART (lower right) were taken, processed, and examined histopathologically by a reviewer blinded to treatment or TG status. WT + vehicle (upper left) reveals characteristic features of cardiac myocytes. WT + HAART and TG + vehicle are essentially indistinguishable from WT + vehicle. In contrast, cardiac myocyte granularity is increased in TG + HAART (lower right). Original magnification, $\times 400$ each; Masson Trichrome.

in TG + HAART after 35 days supports the concept that the mitochondrial toxicity is cumulative and worsens after long-term HAART treatment (Dalakas et al, 1990; Lewis and Dalakas, 1995). This metabolic change reflects the threshold effect seen with deranged oxidative phosphorylation (OXPHOS) in many genetic mitochondrial diseases (Wallace, 1999) and may help to explain poor cardiac performance in TG + HAART after 35 days.

Support is growing for concepts that link altered mitochondrial DNA replication to mitochondrial toxic- ity of NRTI (Brinkman et al, 1998, 1999; Morris and Carr, 1999; Swartz, 1995). Accordingly, elevated plasma LA, a general marker of altered OXPHOS, serves as a laboratory test for mitochondrial dysfunction in AIDS patients treated with HAART (Brinkman, 2000).

Recently, mitochondrial changes were found in hearts and skeletal muscles of Erythrocebus patas fetuses from dams that received relatively low-dose AZT (Gerschenson et al, 2000). In contrast, echocar- 


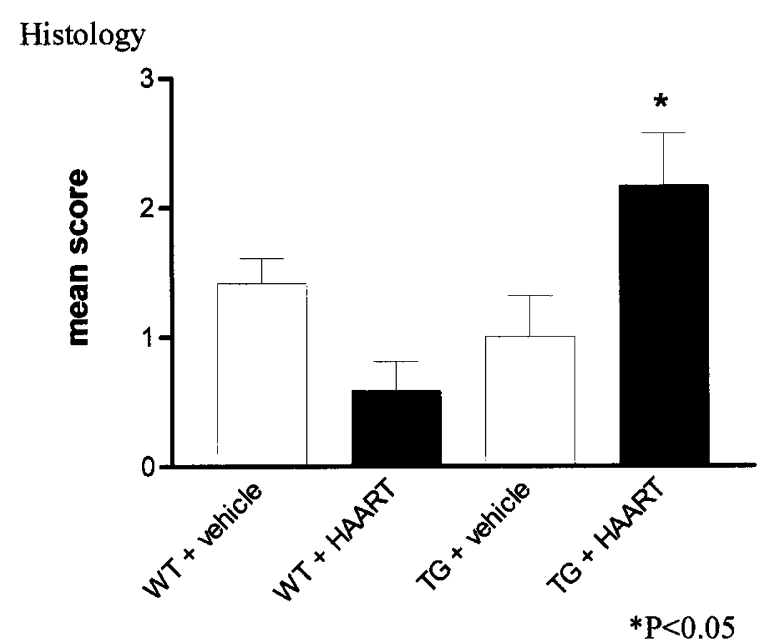

Figure 5.

Histogram of semiquantitative analysis of myocyte cytoplasmic granularity after 35-day treatment. Using a blinded review of slides from each of the cohorts, myocyte cytoplasmic granularity was analyzed. Myocyte cytoplasmic granularity is significantly higher in the TG + HAART cohort than in any other $(p<0.05)$.

diographic changes were essentially absent in another recent study of neonatal patients treated in utero with AZT (Lipshultz et al, 2000). It now appears axiomatic to consider AZT treatment of HIV-1-infected mothers as a potent therapeutic tool to prevent vertical HIV-1 transmission (Lewis, 2001; Mofenson, 2000).

In summary, HAART contributed to structural and functional changes of mitochondrial CM in AIDS TG mice after 35 days. The CM changes were worse than those seen with AZT monotherapy. CM was manifested by increased LV mass (echocardiographically), reversion of ventricular myocyte mRNA expression to a more embryonic phenotype (molecularly), mitochondrial damage (ultrastructurally), and elevated plasma LA (biochemically). After 10 days of HAART, only ANF mRNA was elevated in the TG + HAART cohort.

Taken together, these findings are concordant with the concept that mitochondrial toxicity occurs with HAART and is cumulative (Lewis and Dalakas, 1995). TG mouse models of AIDS coupled with antiretroviral treatment allow us to explore features of AIDS CM and help us to understand pathophysiologic mechanisms of mitochondrial cardiotoxicity from AIDS therapeutics.

\section{Materials and Methods}

\section{Animals and Treatment}

Hemizygous AIDS TG mice were generated from offspring of the colony established by Klotman and colleagues (Kopp et al, 1992). Tg26 is a well-described murine line of NL4-3 $\mathrm{gag} / \mathrm{pol}$ bred on FVB/n background. The TG mouse develops nephropathy (Bruggeman et al, 1997; Kopp et al, 1992) and skin lesions in hemizygotes and severe wasting in the homozygotes (Kopp et al, 1993). For this study, a $2 \times$ 2 protocol included hemizygous TG and WT mice treated for 35 days and 10 days. Cohorts for 35 days included TG + HAART $(n=10)$, WT + HAART $(n=$ 12), TG + vehicle $(n=6)$, and WT + vehicle $(n=12)$. Cohorts for the 10-day protocol included TG + HAART $(n=9)$, WT + HAART $(n=10)$, TG + vehicle $(n=6)$, and WT + vehicle $(n=10)$. All procedures complied with guidelines of and were approved by the Institutional Animal Care and Use Committee.

Each mouse received either drinking water alone or water containing AZT (Glaxo Wellcome, Research Triangle, North Carolina; $0.7 \mathrm{mg} / \mathrm{ml}$ ) plus 3TC (from our Center for AIDS Research, Clinical Pharmacology Unit, Atlanta VAMC, Decatur, Georgia; 0.036 mg/ml) for consumption ad libitum. Volume consumed was determined for individually housed mice. Total and $\mathrm{mg} / \mathrm{kg} /$ day consumption were calculated for each compound. Fresh water was replenished on alternate days. Indinavir (0.9 mg/day; courtesy of Merck Labs, West Point, Pennsylvania) was administered with vehicle (carboxymethyl cellulose $0.1 \%$ in water) by gavage using a curved 20 -gauge stainless steel tube and syringe. 3TC and indinavir consumption were determined similarly. Control animals received vehicle without drug.

\section{Echocardiography of TG and WT Mice with HAART}

Echocardiographic studies were performed in agematched (littermate) WT and TG mice that were treated with or without HAART (35-day or 10-day protocols) in the cohorts described above. Echocardiographic methods were detailed previously (Hoit et al, 1995, 1997; Kadambi et al, 1996; Lewis et al, 2000). Echocardiography was performed by an individual observer who was blinded to treatment or TG status.

Briefly, mice were anesthetized with Avertin (0.25 $\mathrm{mg} / \mathrm{g}$ of body weight) and two-dimensionally targeted M-mode studies (Acuson Sequoia, Mountain View, California) were performed with a $13 \mathrm{MHz}$ imaging and a $7 \mathrm{MHz}$ Doppler transducer. M-mode measurements of end-diastolic dimension (EDD), end-systolic dimension (ESD), end-diastolic posterior wall thickness (EDPWTH), and end-diastolic septal wall thickness (EDSWTH) were made from original tracings. LV mass was calculated as follows: $1.06 \times([E D D+$ EDPWTH + EDSWTH $\left.]^{3}-\mathrm{EDD}^{3}\right)$.

\section{Cardiac mRNA Analysis}

Total RNA was isolated from LV tissue samples from the 35-day and 10-day cohorts, respectively, using the acid guanidinium thiocyanate-phenol-chloroform method (Chomczynski and Sacchi, 1987), as previously described (Lewis et al, 2000). RNA integrity was determined by electrophoresis and quantified spectrophotometrically as 260/280-nm absorbance ratio. Total RNA $(10 \mu \mathrm{g})$ was size-fractionated electrophoretically and transferred to nylon membranes for Northern blotting.

Northern blots were sequentially probed with ${ }^{32} \mathrm{P}$ labeled cDNA probes specific for the SR Ca ATPase (SERCA2, a 2.3-kb cDNA fragment of the rat cardiac 

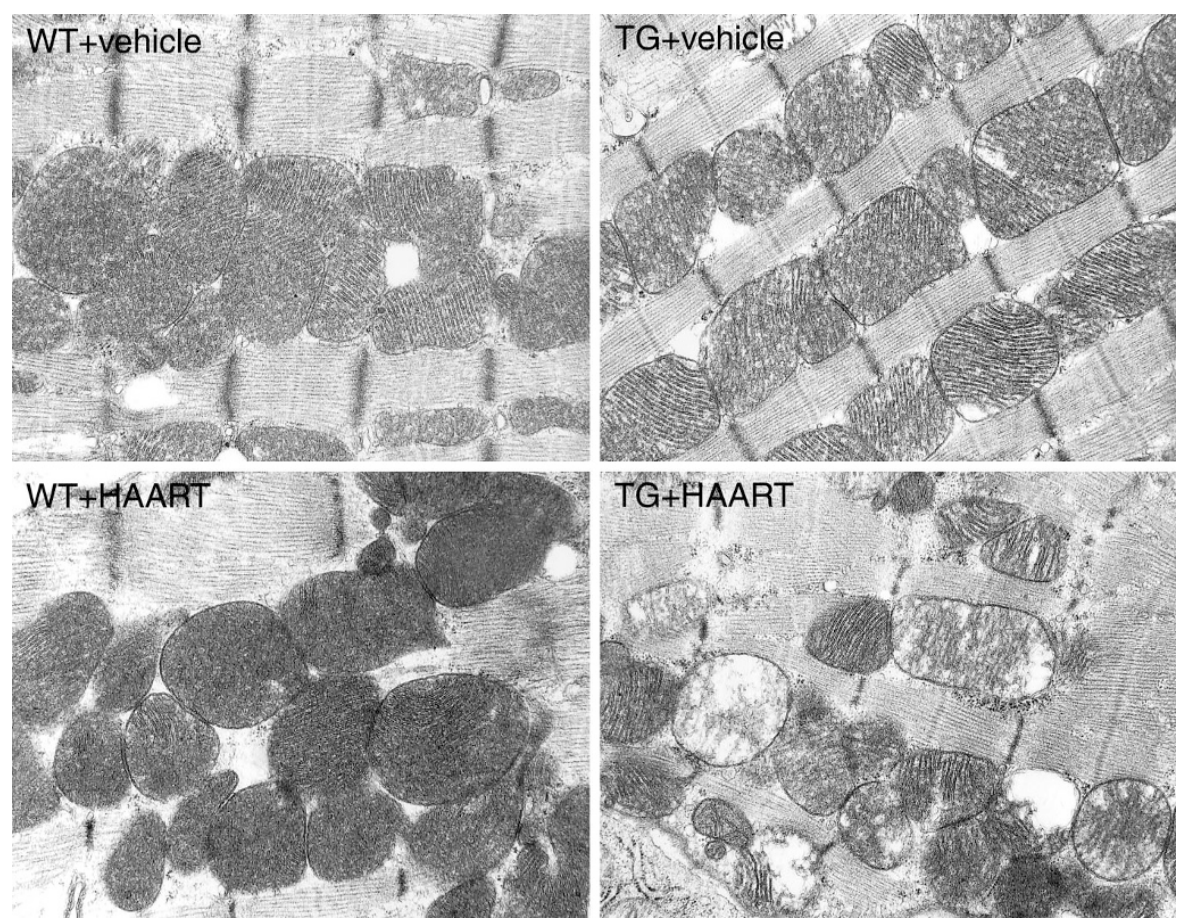

Figure 6.

Ultrastructural changes in cardiac myocytes. LV myocardial samples were processed from WT + vehicle (upper left), WT + HAART (lower left), TG + vehicle (upper right), and TG + HAART (lower right) in the 35-day protocol. In TG + HAART, significant mitochondrial structural damage is found. Changes include cristae dissolution and disruption. Magnification, $\times 14,000$ for each.

SERCA2, provided by Dr. W. Dillmann, University of California San Diego), and ANF (a 0.8-kb cDNA fragment of rat ANF courtesy of $\mathrm{T}$. Inagama, Vanderbilt University, Nashville, Tennessee). Equal loading and uniformity of transfer were insured by normalizing hybridization signal intensity to that of glyceraldehyde phosphate dehydrogenase (GAPDH) mRNA (human GAPDH cDNA, obtained from the American Type Culture Collection, Manassas, Virginia). cDNA probes were radiolabeled and hybridized as previously described (Eleftheriades et al, 1993). The amount of each respective mRNA relative to the amount of GAPDH mRNA was quantified by solid-phase scintillation spectroscopy (Instant Imager; Packard Instruments, Meriden, Connecticut)

\section{Plasma LA}

At termination of the experiments, blood samples were obtained by retro-orbital bleeding of anesthetized TG and WT mice. Plasma was separated, stored briefly at $4^{\circ} \mathrm{C}$, and aliquots (1-4 $\left.32 \mu \mathrm{l}\right)$ were analyzed for lactate colorimetrically using a kit (Sigma Scientific, St. Louis, Missouri). All assays were performed on a Versamax microtiter plate reader (Molecular Devices, Sunnyvale, California) and run in triplicate with external standards with each run.

\section{Histologic Examination}

At termination of the experiments, samples of the hearts were immediately immersion-fixed in neutral buffered formalin. For histopathologic evaluation, each heart slice was dehydrated through a graded series of alcohols, and paraffin embedded (uniformly oriented apex down). Serial sections from the paraffin embedded tissue slice 6 $\mu \mathrm{m})$ were stained with Masson's trichrome. Each slide was examined microscopically by an observer who was blinded to treatment or TG status.

A semiquantitative scale was used for histopathologic comparisons within the experiments. Myocyte cytoplasmic granularity was an index of mitochondrial enlargement and damage in cardiac myocytes in methods analogous to those used in skeletal muscle samples from some mitochondrial diseases (Shoubridge, 1994). Granular and total myocytes were counted in each field examined. Granularity was measured using the following schema: zero granular myocytes per high-power field in ventricular sections was scored as grade zero; one to three granular myocytes per high-power field was scored as grade 1; four to six granular myocytes per high power field was scored as grade 2; seven to nine granular myocytes per high power field was scored as grade 3; more than 10 granular myocytes per high-power field was scored as grade 4. Averages from 10 high-power fields were taken from each slide. Numerical data obtained from this scoring system were statistically analyzed and plotted on a histogram. Photomicrography of slides was performed with a Nikon photomicroscope (Nikon, Garden City, New York).

\section{Transmission Electron Microscopy (TEM)}

A single $2 \mathrm{~mm}$-thick slice was made at the ventricular apex. The LV was identified, immersed immediately in 
a drop of chilled $2 \%$ glutaraldehyde-cacodylate, and sectioned longitudinally and transversely to generate 1 $\mathrm{mm}$ cubes that were further fixed for TEM in $2 \%$ glutaraldehyde-cacodylate for 16 to 24 hours at $4^{\circ} \mathrm{C}$. Each heart sample provided approximately 20 blocks for embedding. Myocardium was rinsed in cold Ringer's solution and post-fixed in $1 \% \mathrm{OsO}_{4}$ (Sigma) in PBS, pH 7.4, for 2 to 3 hours. After osmication and rinses, tissue was dehydrated with graded ethanols, and embedded in Spurr (Spurr, 1969). Myocardial samples were sectioned $(100 \mathrm{~nm})$, stained with uranyl acetate, and examined on a JEOL-JEM-100CX electron microscope (JEOL, Tokyo, Japan). For each embedded sample of myocardium, 10 random sections were selected and photographed at a uniform magnification $(14,000 \times)$. Photomicrographs were enlarged to $5 \times 7$-inch prints and reviewed by a single investigator for structurally abnormal mitochondria. Structurally abnormal mitochondria were operationally defined as those with loss or dissolution of more than $25 \%$ of cristae area (Lewis et al, 2000).

\section{Statistics}

ANOVA was employed to compare data from the different groups.

\section{Acknowledgements}

Kelly Sjögren is acknowledged for processing the manuscript. Bob Santoianni is thanked for thin sections and for excellent TEM photomicrography.

\section{References}

Adrogue HJ and Madias NE (1998). Management of lifethreatening acid-base disorders: First of two parts. $\mathrm{N}$ Engl $\mathrm{J}$ Med 338:26-34.

Arnaudo E, Dalakas M, Shanske S, Moraes CT, DiMauro S, and Schon EA (1991). Depletion of muscle mitochondrial DNA in AIDS patients with zidovudine-induced myopathy. Lancet 337:508-510.

Barbaro G, Di Lorenzo G, Grisorio B, and Barbarini G (1998). Incidence of dilated cardiomyopathy and detection of HIV in myocardial cells of HIV-positive patients. Gruppo Italiano per lo Studio Cardiologico dei Pazienti Affetti da AIDS (see comments). N Engl J Med 339:1093-1099.

Barbaro G, Di Lorenzo G, Soldini M, Giancaspro G, Grisorio B, Pellicelli AM, D'Amati G, and Barbarini G (2000). Clinical course of cardiomyopathy in HIV-infected patients with or without encephalopathy related to the myocardial expression of tumour necrosis factor-alpha and nitric oxide synthase. GISCA. Gruppo Italiano per lo Studio Cardiologico dei pazienti affetti da AIDS. AIDS 14:827-838.

Boubaker K, Sudre P, Flepp M, Furrer H-J, Haensel A, Hirschel B, Boggian K, Chave J-P, Bernasconi E, Opravil M, Rickenbach M, and Telenti A (2000). Hyperlactatemia and antiretroviral therapy in the Swiss HIV Cohort Study (SHCS). Paper presented at 7th Conference on Retroviruses and Opportunistic Infections. San Francisco, California, January 30-February 2, 2000.

Brinkman K (2000). Editorial response: Hyperlactatemia and hepatic steatosis as features of mitochondrial toxicity of nucleoside analogue reverse transcriptase inhibitors. Clin Infect Dis 31:167-169.

Brinkman K, Smeitink JA, Romijn JA, and Reiss P (1999). Mitochondrial toxicity induced by nucleoside-analogue reverse-transcriptase inhibitors is a key factor in the pathogenesis of antiretroviral-therapy-related lipodystrophy (comment). Lancet 354:1112-1115.

Brinkman K, ter Hofstede HJ, Burger DM, Smeitink JA, and Koopmans PP (1998). Adverse effects of reverse transcriptase inhibitors: Mitochondrial toxicity as common pathway (editorial). AIDS 12:1735-1744.

Bruggeman LA, Dikman S, Meng C, Quaggin SE, Coffman TM, and Klotman PE (1997). Nephropathy in human immunodeficiency virus- 1 transgenic mice is due to renal transgene expression. J Clin Invest 100:84-92.

Chaisson RE, Allain JP, Leuther M, and Volberding PA (1986). Significant changes in HIV antigen level in the serum of patients treated with azidothymidine. $\mathrm{N}$ Engl J Med 315: 1610-1611.

Chariot P, Monnet I, Mouchet M, Rohr M, Lefaucheur JP, Dubreuil-Lemaire ML, Chousterman $M$, and Gherardi R (1994). Determination of the blood lactate:pyruvate ratio as a noninvasive test for the diagnosis of zidovudine myopathy. Arthritis Rheum 37:583-586.

Chomczynski P and Sacchi N (1987). Single-step method of RNA isolation by acid guanidinium thiocyanate-phenolchloroform extraction. Anal Biochem 162:156-159.

Church J, Mitchell W, Gonzalez-Gomez I, Boles R, Wetzel R, and Vu T (2000). Near-fatal metabolic acidosis, liver failure in mitochondrial (mt) DNA depletion in an HIV-infected child treated with combination antiretroviral therapy (ART). Paper presented at 7th Conference on Retroviruses and Opportunistic Infections. San Francisco, California, January 30February 2, 2000.

Corcuera Pindado MT, Lopez Bravo A, Martinez-Rodriguez R, Picazo Talavera A, Gomez Aguado F, Roldan Contreras M, Perez Alvarez MJ, Fernandez Garcia A, and Alonso Martin MJ (1994). Histochemical and ultrastructural changes induced by zidovudine in mitochondria of rat cardiac muscle. Eur $\mathrm{J}$ Histochem 38:311-318.

Dalakas MC, IIla I, Pezeshkpour GH, Laukaitis JP, Cohen B, and Griffin JL (1990). Mitochondrial myopathy caused by long-term zidovudine therapy (see comments). N Engl J Med 322:1098-1105.

DHHS (2001). Guidelines for the use of antiretroviral agents in HIV-infected adults and adolescents. Washington: Department of Health and Human Services.

Eleftheriades EG, Durand JB, Ferguson AG, Engelmann GL, Jones SB, and Samarel AM (1993). Regulation of procollagen metabolism in the pressure-overloaded rat heart. J Clin Invest 91:1113-1122.

Gerschenson M, Erhart SW, Paik CY, St Claire MC, Nagashima K, Skopets B, Harbaugh SW, Harbaugh JW, Quan W, and Poirier MC (2000). Fetal mitochondrial heart and skeletal muscle damage in Erythrocebus patas monkeys exposed in utero to 3 '-azido-3'-deoxythymidine. AIDS Res Hum Retroviruses 16:635-644.

Herskowitz A, Willoughby SB, Baughman KL, Schulman SP, and Bartlett JD (1992). Cardiomyopathy associated with antiretroviral therapy in patients with HIV infection: A report of six cases. Ann Intern Med 116:311-313. 
Hoit BD, Khan ZU, Pawloski-Dahm CM, and Walsh RA (1997). In vivo determination of left ventricular wall stressshortening relationship in normal mice. Am J Physiol 272: H1047-H1052.

Hoit BD, Khoury SF, Kranias EG, Ball N, and Walsh RA (1995). In vivo echocardiographic detection of enhanced left ventricular function in gene-targeted mice with phospholamban deficiency. Circ Res 77:632-637.

Hunter JJ and Chien KR (1999). Signaling pathways for cardiac hypertrophy and failure (review). $\mathrm{N}$ Engl $\mathrm{J}$ Med $341: 1276-1283$

Kadambi VJ, Ponniah S, Harrer JM, Hoit BD, Dorn GW 2nd, Walsh RA, and Kranias EG (1996). Cardiac-specific overexpression of phospholamban alters calcium kinetics and resultant cardiomyocyte mechanics in transgenic mice. J Clin Invest 97:533-539.

Kopp JB, Klotman ME, Adler SH, Bruggeman LA, Dickie P, Marinos NJ, Eckhaus M, Bryant JL, Notkins AL, and Klotman PE (1992). Progressive glomerulosclerosis and enhanced renal accumulation of basement membrane components in mice transgenic for human immunodeficiency virus type 1 genes. Proc Natl Acad Sci USA 89:1577-1581.

Kopp JB, Rooney JF, Wohlenberg C, Dorfman N, Marinos NJ, Bryant JL, Katz SI, Notkins AL, and Klotman PE (1993). Cutaneous disorders and viral gene expression in HIV-1 transgenic mice. AIDS Res Hum Retroviruses 9:267-275.

Lamperth L, Dalakas MC, Dagani F, Anderson J, and Ferrari $\mathrm{R}$ (1991). Abnormal skeletal and cardiac muscle mitochondria induced by zidovudine (AZT) in human muscle in vitro and in an animal model. Lab Invest 65:742-751.

Lehman JJ, Barger PM, Kovacs A, Saffitz JE, Medeiros DM, and Kelly DP (2000). Peroxisome proliferator-activated receptor gamma coactivator-1 promotes cardiac mitochondrial biogenesis. J Clin Invest 106:847-856.

Lewis W (2000). Cardiomyopathy in AIDS: A pathophysiological perspective. Prog Cardiovasc Dis 43:151-170.

Lewis W (2001). Absence of cardiac toxicity of zidovudine in infants. N Engl J Med 344:458.

Lewis W and Dalakas MC (1995). Mitochondrial toxicity of antiviral drugs. Nat Med 1:417-422.

Lewis W, Gonzalez B, Chomyn A, and Papoian T (1992). Zidovudine induces molecular, biochemical, and ultrastructural changes in rat skeletal muscle mitochondria. J Clin Invest 89:1354-1360.

Lewis W, Grupp IL, Grupp G, Hoit B, Morris R, Samarel AM, Bruggeman L, and Klotman P (2000). Cardiac dysfunction occurs in the HIV-1 transgenic mouse treated with zidovudine. Lab Invest 80:187-197.

Lewis W, Papoian T, Gonzalez B, Louie H, Kelly DP, Payne RM, and Grody WW (1991). Mitochondrial ultrastructural and molecular changes induced by zidovudine in rat hearts. Lab Invest 65:228-236.

Lipshultz SE, Easley KA, Orav EJ, Kaplan S, Starc TJ, Bricker JT, Lai WW, Moodie DS, Sopko G, Mclntosh K, and Colan SD (2000). Absence of cardiac toxicity of zidovudine in infants. Pediatric Pulmonary and Cardiac Complications of Vertically Transmitted HIV Infection Study Group (see comments). N Engl J Med 343:759-766.
Lonergan JT, Havlir D, Behling C, Pfander H, Hassanein T, and Mathews WC (2000). Hyperlactatemia in 20 patients receiving NRTI combination regimens. Paper presented at 7th Conference on Retroviruses and Opportunistic Infections. San Francisco, California, January 30-February 2.

McCurdy DT 3rd and Kennedy JM (1998). AZT decreases rat myocardial cytochrome oxidase activity and increases betamyosin heavy chain content. J Mol Cell Cardiol 30:19791989.

Miller KD, Cameron M, Wood LV, Dalakas MC, and Kovacs JA (2000). Lactic acidosis and hepatic steatosis associated with use of stavudine: Report of four cases. Ann Intern Med 133:192-196.

Mofenson LM (2000). Perinatal exposure to zidovudine: Benefits and risks. N Engl J Med 343:803-805.

Moore R, Keruly J, and Chaisson R (2000). Differences in anion gap with different nucleoside RTI combinations. Paper presented at 7th Conference on Retroviruses and Opportunistic Infections. San Francisco, California, January 30February 2, 2000.

Morris AA and Carr A (1999). HIV nucleoside analogues: New adverse effects on mitochondria? Lancet 354:1046-1047.

Ruprecht RM, O'Brien LG, Rossoni LD, and NusinoffLehrman S (1986). Suppression of mouse viraemia and retroviral disease by $3^{\prime}$-azido-3'-deoxythymidine. Nature 323:467-469.

Shoubridge EA (1994). Mitochondrial DNA diseases: Histological and cellular studies. J Bioenerg Biomembr 26:301310.

Spurr AR (1969). A low-viscosity epoxy resin embedding medium for electron microscopy. J Ultrastruct Res 26:31-43.

Swartz MN (1995). Mitochondrial toxicity: New adverse drug effects (editorial and comment). N Engl J Med 333:10991105.

Ter Hofstede H, De Marie S, Foudraine N, Danner S, and Brinkman K (2000). Four cases of fatal lactic acidosis due to mitochondrial toxicity of NRTI treatment: Analysis of clinical features and risk factors. Paper presented at 7 th Conference on Retroviruses and Opportunistic Infections. San Francisco, California, January 30-February 2, 2000.

Wallace DC (1999). Mitochondrial diseases in man and mouse. Science 283:1482-1488.

Yarchoan R, Klecker RW, Weinhold KJ, Markham PD, Lyerly HK, Durack DT, Gelmann E, Lehrman SN, Blum RM, and Barry DW (1986). Administration of 3'-azido-3'deoxythymidine, an inhibitor of HTLV- III/LAV replication, to patients with AIDS or AIDS-related complex. Lancet 1:575580. 